\title{
Téoros
}

Revue de recherche en tourisme

\section{Quand le touriste rencontre l'homo faber}

\section{Marc Laplante}

Volume 15, numéro 2, été 1996

Patrimoine industriel

URI : https://id.erudit.org/iderudit/1075021ar

DOI : https://doi.org/10.7202/1075021ar

Aller au sommaire du numéro

Éditeur(s)

Université du Québec à Montréal

ISSN

0712-8657 (imprimé)

1923-2705 (numérique)

Découvrir la revue

Citer cet article

Laplante, M. (1996). Quand le touriste rencontre l'homo faber. Téoros, 15(2),

5-8. https://doi.org/10.7202/1075021ar d'utilisation que vous pouvez consulter en ligne.

https://apropos.erudit.org/fr/usagers/politique-dutilisation/ 


\section{f \\ QUAND LE TOURISTE RENCONTRE L'HOMO FABER}

\author{
Marc Laplante, professeur \\ Département d'études urbaines et touristiques \\ UQAM
}

* Hono fabers (l'homme fabricatear). "Nous croyons qu'il est de l'essence de l'honine de créer matériellement et moralement, de fabriquer des choses et de se fabriquer luimême. Homo faber, telle est la definition que nous proposons". . Le philosophe $H$. Bergson, qui donne cette définition, l'oppose à celle d' "hono sapiensw ("né de la réflexion de l'homo faber sur sa fabrication") et à celle de l'whono loquaxs ("dont la pensée, quand il pense, n'est qu'une réflexion sur sa parole").

L'objectif de cet article est d'essayer de comprendre pourquoi et comntent cet homo faber est devenu une attraction touristique pour les touristes de sociétés post-industrielles. Le processus qui conduit de l'homo faber au travail sacralisé en tant qu'attraction touristique ne peut se comprendre que par une brève excursion en sociologie da travail.

\section{LE TRAVAIL DANS L'HISTOIRE HUMAINE}

Un tel sujet se prête mal à un résumé en quelques lignes. Il faudra emprunter des raccourcis pour les besoins de ce texte: l'idé principale à saisir à ce propos est le chambardement du monde du travail qui $s^{*}$ opère en peu de temps avec la première révolution industrielle au début du XVIIle siècle en Europe.

Avant l'industrialisation ${ }^{2}$, le travail mobilise l'énergie des humains des deux sexes et de tous les âges; il failt partie des conditions de survie. Les savoir-faire sont acquis par essais et erreurs sur de longues périodes et constituent l'essentiel de l'héritage transmis d'une génération à une autre.

Au plan culturel - celui qui nous innorte davantage pour cette analyse - le travail n'est pas survalorisé par rapport aux autres activités humaines même s'il requiert beaucoup de temps et d'énergie. L'homme ne se définit pas par son activité produc= trice mais plutôt par sa capacité de vivre en société et de se conformer aux normes et aux valeurs qui ticnnent ensemble les collectivites humaines,

L'industrialisation commence dans le contexte de l'aflimation des pouvoirs de la raison sur le monde et la vie. Le développement des connaissinces et des méthodes pour les acquérir ont convaincu l'homme moderne qu'il peut tout taùre par lui-même, que tout $s$ explique par la pensée, la logique ou le calcul sans recourir aux sor= ciers, ou aux autres incarnations de pouvoirs cachés, mystếrieux et incontrôlables. Du XVe et au XIXe siècle, la science s'installe comme puissance dominante après ses premières conquêtes qui ont favorisé les découvertes de nouveaux continents, la maîtrise plus grande des sources d'énergie, le rejet des enseignements de la tradition quat aux origines de l'honme, à la place de notre planète dans le système solaire et surtout, quant aux dangers de briser la continuité des savoir-faire elaborés sur plusieurs siècles passés.

Brutalement, avec les premières manufactures, le monde du travail change sur tous les plans: il est séparé du reste de la vie, il s'échange contre des biens et de l'argent, il est fractionné en tâches spécifiques dont l'apprentissage est rapide, etc. Nous retiendrons surtout deux traits du nouveau contexte créé par l'industrialisation: les machines assurent une production stan= dardisée et augmentent considérablement la productivité par travailleur; ce demier devient de plus en plus le serveur de ces machines. De nouveaux savoir-faire naittront, très nombreux, dans les rapports hommes-machines; ils seront inventés par les ingénieurs et enseignés aux travailleurs; des écoles apparaîtront par la suite pour acquérir ces nouvelles compétences techniques; la recherche scientifique battra le rythme accéléré des révolutions technologiques et des changements dans la formation des travailleurs. Enfin, en lien direct avec ce quil précède, se formeront des classes sociales et commenceront des luttes entre ces classes fondées sur les positions antagonistes entre employeurs et employess, entre propriétaires du capital et salariés, entre les travailleurs du secteur primaire, longtemps laisses pour compte des révolutions technologiques, ceux du secteur de la transformation et les nouvelles classes des services. S'ensuivra un gigantesque mouvement entre secteurs mais aussi, mouvement des campagnes vers les villes, des pays moins industrialisés vers d'autres qui le sont plus, etc. 
Le taylorisme du début du XXe siècle est donné souvent comme le paroxysme de la dépossession de l'homme par son travail et de son aliénation modeme: l'ingénieur Taylor étudie scientifiquement les gestes les plus efficaces de l'homme au service des machines. L'ergonomie naîtra plus tard pour savoir comment ne pas trop épuiser la machine humaine au travail mais Taylor se préoccupait déjà de ne pas gaspiller la force de travail comme on l'avait fait depuis la première manufacture: entre celle-ci et les premières chaînes de montage des automobiles, le sprix d'un travailleur avait passablement augmenté: mieux valait alors arationalisers ses gestes pour le rendre plus productif et moins socialement coûteux. (Aujourd'hui, on emploie le même mot pour justifier sa mise à pied, les machines pouvant fonctionner sans luil)

En cette fin du XXe siècle, la condition générale de l'ouvrier rivés à ses machines s'est clargie à beaucoup d'autres travailleurs: à ceux de la plupart des industries du secteur primaire et surtout, aux très nombreux employés du secteur tertiatire asservis à des tâches répétitives et lićes à d'autres machines tout aussi éreintantes (ordinateurs, par exemple). Certaines conditions nouvelles de travail ne doivent pas nous illusionner: horaires variables, congés mobiles, travail à temps partiel, à la maison, etc, n'enlevent pas l'obligation de travailler, ne laissent pas plus d'autonomie à l'acte productif, ne facilitent pas la mobilité professionnelle et surtout, elles réduisent la sécurité d'emploi, les revenus du travail et la valeur même de celui-ci.

Dans la culture post-industielle, la division drastique entre travail et non-travail tend à disparâtre. En présence de tant de gens qui n'ont pas de travail ou ne travaillent plus, ceux qui sont au travail se disent uchanceuxs de travailler! En tait, pour beaucoup, la chance consiste à avoir des revenus supérieurs à ceux qui dépendent de $1^{+}$assurance-chömage, de la sécurité du revenu ou des allocations du abien-êtres social. Certains ont peut=être plus de uchancess en travaillant au noir pour arrondir les allocations et autres assurances mais rien de tout cela redonne au travail si place dominante dans les valeurs modertes.

Il y a moins d'un demi-siecle encore, tratvailler était donne comme une vertu gat les employeurs, bien sûr, mais a aussi par foutes les institutions du pouvoir qui y voyaient lá source de l'ordre social et du progres. église, justice, maisons d'enseignement, etc. Aujourd'hui, même les employeurs n'ont plus d'arguments séduisants pour démontrer les vertus du travail. On leur demande de créer des emplois pour activer les flux économiques et financiers. Euxmêmes souvent sont maintenant asservis à des supersystèmes de production opérant sans frontières. Alors, travailler...

\section{LE TOURISTE ET L'HOMO FABER}

Le touriste est largement un "produits de la société industrielle et davantage encore, de la culture post-modeme? Il est aussi un travailleur qui a quitté monentanément son activité productive pour aller vers d'autres environnements. Et, en vacances, parmi ses interêts, il s'occupe du travail d'autres hommes ailleurs. Dean MacConnell' woil dans cette pratique touristique un des nombreux paradoxes qui jalonnent l'histoire du tourisme. Quand on y refléchit, quand on saisit un tant soit peu les changements survenus dans le monde du travail depuis deux siècles, fatut-il s"étonner de wir le trawaillèr d'aujourd'hui s'interesser à l'homme fabricateur durant ses woyages de vacances?

II est de I'essence de Ithomme de créer materriellement et movalement, de fabriquer des choses et de se fabriquer lui-mêne, selon les philosophes. La condition génerale du travailleur d'aujourd'hui conespond-elle à celle de l'honto faber? Pour quelques-uns certainement mais, pour l'immense majorité, le travaill ne vaut plus que par ce qu'il rapponte en argent edou en prestige, Combien de travailleurs peuvent se clire maâtre du processus qui va de la cueillene des matiêres premières à la wente d'un produit fini dans lequel on retrouvera leur sivoir-faire, leurs latents de créateurs et donc, l'expression d'cux-mênes? Celte situation est si rare maintenant que nous désignons de noms spéciliques ceux et celles qui la vivent: atrisans, pratiquatis d'un métier d'art, clc. Parmi les chumps reconnus des activitês de temps libre, on trouve celui du bricoleur, personne gui essaie de produire une chose avee peu d'outils, et peu de matiere première. Au Québec, on a déjàt nommé spatenteux ces bricoleurs qui manifestaient une certaine cráliviế pour assembler, en les recyclant, divers objets de la vie quotidiennes. Il faut voir dans ces pratiques des tentatives pour retrouver l' homo faber perdu.

Mais l'intérêt pour de telles retrouvailles s'exprime aujourd'hui de très nombreuses façons. Une simple énumération de celles-ci nous amènera à décrire certains comportements touristiques manifestant ce même intérêt:

expansion rapide et soutenue des loisirs scientifiques et techniques, de l'horticulture à l'astronomie;

recherches patrimoniales, mise à jour et mise en exposition d"anciennes manieres de produire, de savoir-faire ingénieux, de genese des modes de fabrication, de l'histoire des outils, de celle de la maîtrise de l'énergie, etc:; missance et enrichissement de grands musćes modemes des sciences et des technologies;

- multiplication des sauvetages de quartiers industriels, d'industries;

interprestation des travailleurs et de leurs conditions de vie dans de nombreuses branches d'activités:

crétion, plus récente, d'ccononusces où le visiteur woit la production en cours et peut acheter le produit fini;

festivals ou événements socio-culturels mettant en vedette des peintres, des sculpteurs ou d'autres artistes ou artisans en train de créer.

Celte énumération reste incomplète mais elle exprime suflisamment une grande tendance dans la culture posi-moderne: faire du travail une exposition, une sorte de mise en vitrine comme un etalage d'un grand magasin. A mesure que le travail perd son sens historique de manifestation de savoir-faire, à mesure que les oeuvres modernes de création et de haute technologie se font dans les lieux clos des haboratoires et des centres d"experimentation, à mesure se développent dans la culture premièré des goûts et des intérêts pour la pratique artisanale (bricolage, auto-production, apprentissage des sawoir-fäire anciens, etc.) et pour la découverte par l'observation d'habiletés manuelles, de techniques de production, d'exploits scientifiques et techniques, etc.

Pourguoi cette tendance prend-elle tant d'ampleur en cette fin du XXe siècle? Pour retrouver des racines, des origines, pour sauver de l'oubli des choses essen- 


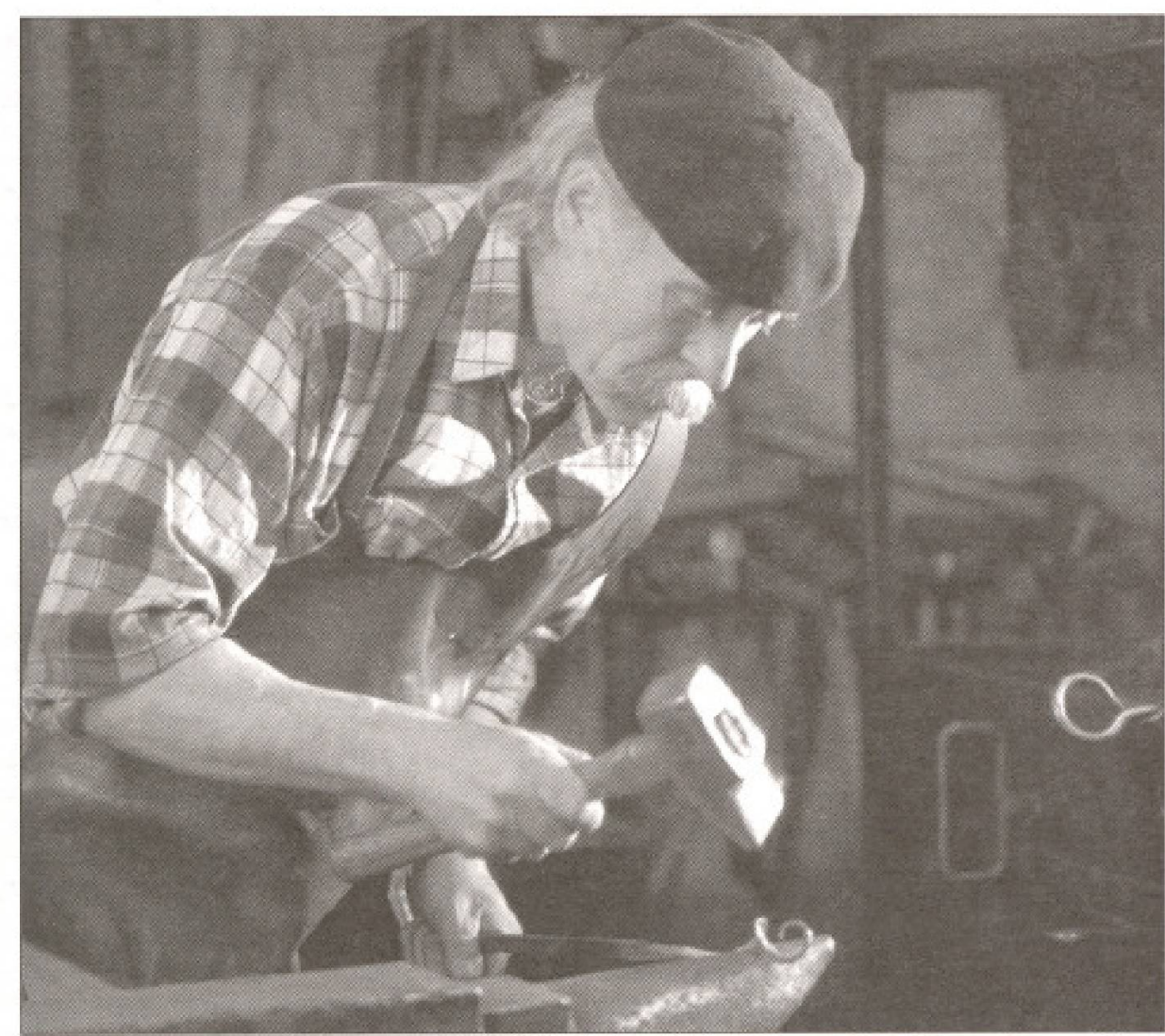

Gry Bel, forgeron d'art. Economusée de la fonge, lle d'Orláans. (Source C. Dufour, photographe).

tielles? On regarde peut-être ainsi dans le

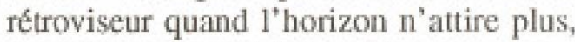
quand la route suivie semble mener vers l'inconnu et l'incertain (chômage croissant, automation rapide, eclatement du travail organise, accroissement des gens à la retraite, incitation à cesser de travailler, entrée tardive et difficile sur le marché du travail, impossibilitế $\mathrm{d}^{\dagger} e ́$ laborer des plans de carrière, etc.). Il faudrait, à ce propos, résumer de nombreux travaux récents tentant de comprendre l'intérêt croissant pour le patrimoine en général et, pour notre propos, le patrimoine industriel. Quelques références bibliographiques aideront le lecteur intéressé à approfondir cette question?

Considérant ce qui précède, il n'est plus surprenant de constater que le patrimoine industriel et l'observation de l'hono faber en train de fabriquer fassent partie des intérêts et des pratiques des touristes. Ces derniers sont des travailleurs qui, très souvent, ne se réalisent plus en travaillant. Ils appartiennent à des sociétés très avancées au plan des sciences et des techniques où se développe une nostalgie de l'homo faber, comme si, en chacun de ces travailleurs modemes, sommeillait toujours cet homo faber qui n'a plus les moyens, les sawoir-faire pour se manifester.

Qu'importe-t-il de savoir maintenant sur ce type de touristes et sur les interventions de responsables touristiques pour les satisfaire?

Il importe d'abord de rappeler que $1^{*}$ attention du touriste en présence de l'homo faber a l'oeuvre n'es pas nouvelle. Dans les récits de voyageurs des XVIIIe el $\mathrm{XIXe}$ siécles, on relève fréquemment des descriptions détaillées de techniques de production, d'outils et d'ingéniosité pour s'en servir, d'oeuvres typiques qui tont ha joie des collectionneurs, etc.

Toujours et universellement, croyonsnous, les manifestations de l'habileté manuelle, du gếnie accunulé par des prat tiques ancestrales et bien conservées, ont fasciné les woyageurs. Les touristes, sitôt nés, ont connu celte faxcination,

Plus récemment, depuis un quart de siècle environ, le tourisme intemational de masse a amené des millions de gens des societes hautement technologiques vers des pays en voie de développement. Sans faire d'efforts particuliers, ces touristes pouvaient ainsi se trouver en présence d'authentiques homo faber, observer leurs processus de production et acheter leurs produits.

Il faut savoir à ce propos que ces sociétés dites en voie de développement sont des milieux qu'on pousse a l'industrialisation comme seule voie de solution à leur pauvreté, que celle-ci est bien relative parce qu'on la confronte à la richesse et au bien-être matêriel des sociétés industrialisées, et qu'en fait, il s'agit de sociétés traditionnelles ou pré-industrielles comme il en a existé partout sur la terre jusqu'au XVIIIe siecle.

En société traditionnelle, le travail ne se fait pas en usine, pas plus d'ailleurs que l'éducation: les champs cultivés ne sont jamais loin du coeur des villages et des villes, les marchés publics sont permanents, nombreux et diversifies et on $y$ produit sur place beaucoup de biens, les métiers s"exercent sur le pas des portes ou à côté des maisons, la production est constante car on ne fait que très peu de stockage, l'acheteur est souvent en présence du fabricant, etc.

Les touristes qui parcourent ces sociétés ont fréquemment sous leurs yeux des homo faber en train de produire. Si le produit se fait en peu de temps, ils peuvent en suivre la réalisation; dans d'autres cas, leurs visites les conduiront dans des quartiers urbains ou dans des regroupements de travailleurs en un lieu donné où il leur sera possible de suivre, une érape après l'autre, tout un processus de production".

Parallèlement, dans les sociétés postmodernes, le patrimoine industriel et les réalisations les plus impressionnantes de la science et de la technologie sont muséifies: musées de la science et de la technologie, visites industrielles organisées, reconstitution, restauration et ouverture aux visiteurs de sites industriels anciens, concours et expositions d"inventeurs, d'amateurs de loisirs scientifiques et toutes les autres façons mentionnées ci-haut de favoriser les retrouvailles du travailleur actuel avec l'homo faber et ses oeuvres. Chez lui, comme en voyage d'agrément, l'intêrêt frour l'art et le savoir-taire des afabricateursw, au sens ancien de ce mot", est constamment nourri et satisfait. 
Satisfait? L'expression est sürement trop forte. La museification de cette ressource a conservé l'esprit principal du musée dans de nombreuses occasions: instruire, éduquer, éveiller les consciences. Parfois, on fait des concessions à l'air du temps en introduisant quelques machines pour l'interactivité, machines qui se caractérisent par leur aptitude à tomber en panne rapidement...! Aussi longtemps que ces mises en exposition sont faites pour les résidents, ceux-ci peuvent prendre le temps voulu pour les apprécier, quitte à revenir sur les lieux occasioninellement.

Il en va tout autrement pour les touristes parce que les sacralisations touristiques des sites et lieux d'exposition de l'homo faber et de ses oeuvres sont rares ${ }^{10}$. Les responsables de l'offre touristique savent maintenant que beaucoup de touristes sont intéressés à ce sproduits (le patrimoine industriel et la technologie de pointe) mais ne fabriquent pas ledit pro= duit et ne font que renvoyer leurs clients aux réalisations des responsables du patrimoine. Quand ces derniers sont conscients de leurs clientèles touristiques ef de leurs besoins particuliers, les touristes $y$ trouvent leur compte. Mais on peut compter facilement les opérations réussies dans ce domaine".

Moins structurées, plusieurs activités reliées plus ou moins directement à l'homo faber retiennent les touristes sur leurs parcours. Si le patrimoine industriel muséfié expose surtout les oeuvres de l'homo faber. quand celui-ci est au travail dans, les lieux publics ou dans ceux qu'on ouvre aux touristes, ses activités attirent l'attention comme celle du magicien amuseur public. Autour des artistes (faut-il mettre des guillemets dans ce cas?) peignant sur les places publiques, dans les vieux quartiers urbains, ou dans Charlevoix, en Gaspésie ou dans les Laurentides (liẹx déjảa fréquentés par des artistes reconnus), des touristes s'attardent, font des photos, achètent parfois des oeuvres à titre de woulvenirsw, etc. II en est ainsi pour l'action en public de d'autres artisans et de fabricants utilisant de vieux sawoir-faire: forgerons, tisserands, sculpteurs, etc. Lintérêt pour les centres d'interprétation s' accrôt quand ceux-ci laissent voir des processus anciens de fabrication ${ }^{12}$. Ce même intêrêt fournira de bonnes clientèles aux visites industrielles si celles-ci permettent de suivre les étapes de production du début à la fin et de comprendre ce qui se passe, notamunent, tous les savoir-taire réunis pour obtenir un produit ouvré. Il suffit parfois de bien démontrer wcomment ça marches pour attirer les visiteurs ${ }^{13}$. En fait, on pourrait sicraliser de très nombreuses productions, y compris celles qui fournissent des biens communs de la vie quolidienne: le parfum, le chocolat, le vin, la bière, etc. Ceci sera encore plus intéressant si le touriste peut voir faire un produit dit typique d"un pays, ou d $\mathrm{d}^{+}$un groupe culturel (les parfums ou les vins en France, le chocolat en Belgique, la biềre en Allemagne ou en République tchèque, le coca-cola aux États-Unis, etc.) et, si possible, l'essayer ou le déguster.

Pour rester dans l'espril de ce lexte, concluons que les createurs de produits touristiques ont benucoup de prin sur la planche pour rendre touristiquement intéressint un vaste ensemble d'activités humaines qui ont permis à l'homme, depuis toujours, de se fabriquer lui-même. Ils n'oublieront pas cependant de s'associer aux connatsseurs en patrimoine pour metre en valeur la ressource essentielle: la précieuse habileté des gestes humains qui contiennent une large part de notre mémoire commune.

\section{NOTES ET RÉFÉRENCES}

1 Citation de H. Bergson: "La pensée ef le mouyant", p. 105, donnde dins André Lalande (1972) Wrobulawe technigne ef criligue de la philosolnhic, Paris, PUF, p. 418.

2 Pour un expogé plus rigoureus des chungements sociaux et culturels tésultant de l'industrialisation, on pourru consulter: Mare Laplante (1996): Lexperience fomrishigue

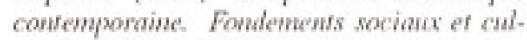

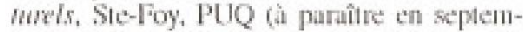
bre), chapitre 2: "Socité al culture de l'Oxidenal indusunialis.".

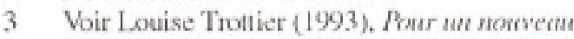

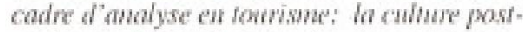
makerne, UQAM, ménoire de muärise en sociologie.

4 Dean Muconnell (1976), The Towis, NY, Shocken Books, p. 36. "Latwor Iransforms taw material into useful objects. Modemity is transfoming lathor into cultural production attended by wourists and sightseds who ance moved by the universality of work relations not as this is representeal through theit own wotk (from which he is alienalted), but as it is wealed to them it their lessure through the displayed wotks of others."

5 Raymonde Lamotic Louise de Grostogis, Lis: Nantel (1974). Les patenteux du Qubere, live a l "origine d"un atuliovisted de I'ONE (1974) ayant le mểme tilre (problucteur delegué: Claude Ineflily) Livn pary ches Pati Pris, Montral, 1974, $272 \mathrm{p}$.
6 Lexpression: culture premiêre est empruntéc a Fernand Dumont (1987): Le son de la culture, Montréal, l'Hexagone. Elle réfere ì la cule vécue au quotidien, à celle apprise par la premiere socialisation: culture informelle faite de passions et de sentiments plutố que de raison et de savoir, cte. L'auteur l'oppose à la eulture institutionnalisce,e, appeléte aussi culture seconde, savante, etc. Les optritions de muséfication du patrimoine industriel par l'Etat appartiennent aux manifestations de cette culture institutionnalise.

7 On consultera un des ouvrages suivants qui peuwent aider à comprendre l'intétêt pour le patrimoine dans les sociétés post-industrielles.

- Roland Schaer (1993): Linvention des musées, Panis; Gallimard.

- François Choay (1992): L'allegorie du patrimoine. Paris: Seuil.

- Jacques Mathieu (1986) (sous la dir. de): Ende de la construction de la ménoire collective des Ouebecois an $X X \bar{c}$ sidcle. Québec: CELAT, Université Laval.

- Jean Duvallon (sous la dir. de) (1986): Claquenurer pour ahsi dire toul l'univers: la mise en exposition. Paris: Centre de création industrielle, Centre Georges Pompidou.

- Alain Bourdin (1984); Le parrimoine réinvente. Paris: PUF.

8 Dans un quartier du vieux Fez, au Maroc, les artisans du cuir procodent à toutes les operations traditionnelles pour produire les cúdebres cuirs marocains. Les touristes peuvent observer ces travailleurs du haut de quelques belvédères. C'est un lieu riche en couleur (les teintures) mais aussi en odeurs...

9 Selon le Robert, wfabricateurw était jadis synonyme d'artisan; aujourd' hui, ce dernier mor désigne généralement un travailleur qui produit dans l'esprit des artistes; fabricateur est une expression pejorative: falsification de fausse monnaic de faux papiers: pourtanl quel savoir fuire souwent ther ocs gens! Curieux tout de même, ce glissement de sens..

10 La sacralisation est un processus de mise en exposition spécialisé qui tient oomple du statut propre du touriste et du contexte particulier qui encadre ses expériences de dboouvertes. Voir Marc Laplante (1996): op. cit., chapitre 5.

11 Au Qubhe, il faut mentionner au moins le Musce de la Civilisation à Québec el le Centre d"Archéologie ef d"Histoire de Pointea-Callieres oomme des institutions qui se sont crefes en pensant aux touristes. L'howo fober of ses ocuvres sont parfois révelts aux visiteurs. Mais l'Association québócoise pour le patrimoine industriel prend maintenant plusieurs initiatives pour que les tounistes comme les résidents trouvent gatisfaction à frếquenter ce genre d'attractions.

12 Pat exemple, l'expérience de l'économusé de la papterie Saint-Gilles a saint-Josephde-la-Rive, en Charlewoix.

13 Pur exemple, voir fonctionner de près une turtine en action a Manic 5 ou a la Centralc de Besuharnois, ecomprendre le principe de l'Élicno à Cap Chat, etc. 